\title{
Applying Classifications Techniques in Job Recommendation System for Matching of Candidates and Advertisements
}

\author{
Gözde Özcan, Şule Gündüz Öğüdücü \\ Istanbul Technical University, Turkey
}

\begin{abstract}
In this paper, a reciprocal job recommendation system, CCRS (Classification - Candidate Reciprocal Recommendation), is proposed. With this proposed system, offering job advertisements in a sequence for candidates that they can get feedback reciprocally by using the user's profile, interaction and preference information is aimed all together. An approach based on the preference information of the candidates has been used to determine the rankings of the jobs in the recommended list, the interaction information of the candidates and the Trust Rank method has been used for eliminating the data sparsity caused by the unknown feedback values of the jobs, and the success of different classification methods has been compared to estimate the feedback rate of the advertisements for the target candidate. CCRS also addresses the cold start problem of new candidates joining the site by providing recommendations based on their profiles. The performance of the proposed method was evaluated by using various performance measurements on an actual data set received from an online recruiting website. Evaluation results show that the proposed method outperforms the compared methods for the top 10 ranked recommendations.
\end{abstract}

\section{Introduction}

Excessive information has been generated with the increase of the number of users on online social networks and it has become difficult to find and to decide the items users can choose among them.

Many websites have developed recommendation systems to help them find the items that could interest their users to overcome this problem [1]. The aim of this system is to recommend items that might interest users, such as: books, movies, pharmacy products and restaurants. There are different approaches used for these recommendations; such as content-based [3], collaborative filtering [4] and hybrid methods[5].

A special class of recommendation systems is also reciprocal recommendation systems. These systems are designed for practices focused on people-to-people recommendations and include recommendations that ensure satisfaction of both sides. Reciprocal recommendation systems are different from traditional userxitem recommendation systems. While traditional recommenders only recommend products according to the preferences of the person for whom the recommendations are generated, reciprocal recommendation systems recommend people-to-people according to the preferences of both sides [6]. Many systems include or use reciprocal recommendation systems. One of the most important examples is the job search website. These sites aim to help jobseekers find jobs that match their preferences and characteristics or recommend reasonable candidates that match their needs to recruiters [2].

The basic problem for reciprocal recommendation is to make a match that fulfils the mutual needs of both users. The relations between the users must be modeled considering the preferences of both sides. In this study, a reciprocal job recommendation method that handle the users' profiles, interaction features and preferences all together to make an recommendation for the candidate is presented. A summary of the contributions made by this model are below:

- A model using profile, preference and interaction information of both sides for reciprocal job recommendation is proposed. This system recommends a job list to the target candidate by using the applications made by the candidates that are similar to the target candidate and the preference information of these jobs.

- In the proposed system, two labeling methods are proposed that use candidate interactions information and Trust Rank method in order to eliminate lack of data due to lack of positive/negative response information to the candidates.

- As a part of the proposed system, an approach based on the preference information of the candidates for the sequencing of the recommended job list is used.

- The proposed system has been evaluated by using a data set received from a job recruiting websites.

An important advantage of the proposed method is to avoid cold start problem for the candidates. With this method, there is no need for the candidate to make any entry other than profile information, to make a recommendation for the new candidate that signs up the system. 
The rest of this study was organized as follows: Section 2 presents a literature review about reciprocal recommendation. The 3 rd part includes the description of data set and the problem, the 4th part provides the structure of the proposed system, the 5th part provides the description of the methods used for the proposed systems, the 6th part presents the experimental results. Finally, section 7 contains some conclusions and some idea about our future work.

\section{Related Works}

Reciprocal recommendation systems offer recommendation according to the users' mutual preferences. There are different application areas for the reciprocal recommendations, such as: online recruiting services, online dating services, online mentoring systems. Recommendation systems are basically divided into 4 categories[7]: collaborative filtering, content-based, information-based and hybrid approaches. A lot of research has been done for recommendation systems in academic and industrial fields using these approaches. The concept of reciprocal recommendation is first introduced in [2]. In this study, the characteristics of reciprocal recommendation systems have been analyzed in detail and compared with the traditional recommendation systems.

[8]iHR+ method offers reciprocal recommendations for mobile applications. This method divides the profile information of job seekers and employers into 2 categories: self-description and preferences. It generate recommendation using crosssimilarity among users' mutual characteristics. A content-based approach that uses the skills of the candidates and the requirements of employers to propose suitable candidates for a specific position is presented in [9]. But these two methods did not take the users' preference information and the interaction characteristics into account when generating recommendation. [1] proposes a new collaborative filtering method to improve user recommendations in reciprocal and bipartite social networks. This proposed method has been evaluated for online dating networks. However, this method did not use the content information to generate recommendation. [6] CCR method presents a new reciprocal contentcollaborative based recommendation system for online dating systems. While the content-based part finds the similar users, the collaborative filtering based part uses the interaction of the similar users to produce reciprocal recommendations. This system also avoids the cold start problem.

Apart from the above mentioned methods, our recommended method proposes the content along with interaction and preference information of both sides. Unlike other methods, for the users' feedback information, it offers an approach that handles the preferences of the jobs with classification methods and estimates the feedback rate for the target candidate. Thus, the content and preference information for both users are used together to obtain the information of whether they would prefer each other. At the same time, the proposed method avoids the cold start problems of the candidates and the data sparsity problem that caused of the lack of feedback information of candidates. Another difference from other methods is that it uses a different approach that uses the preferences of the candidates to sequence the recommendation list of jobs.

\section{Definitions and Problems}

This section includes the contents of the data set used, and the definition of the problem for which solutions are proposed.

\subsection{Data Introductions}

The proposed method was generated on a data set received from Kariyer.Net[10], a job seeking website. Data received from this website consist of information not containing the identity and contact information of users. This data sets consists of 7455 applications in total, for 10 job postings by 5600 candidates between April 2015 and December 2015.

\subsubsection{Candidate profile features}

Candidate profiles consist of 19 features. Those items are listed below:

- The Curriculum Vitae Status: Divided in 2 groups: True(Active) or False(Passive).

- Marital status: Candidate's marital status. Divided in 2 groups: Single or Married.

- Gender: Candidate's gender. Divided in 2 groups: Male or Female.

- Age: Candidate's age. Between 15 and 58.

- Military state: Candidate's military state. Divided in 5 groups: Continuing, Privileged, Done, Probation, Not Done.

- Educational Status: Candidate's academic degree. Which consist of 6 groups: Doctorate, Postgraduate, Undergraduate, Technical School, High School, Primary School.

- Driving license: Candidate's driving license class info. Divide in 2 groups: True(has) or False(has not).

- Disability Status: Candidate's disability status. Divide in 2 groups: True(disabled) or False(not disabled).

- Country: The Country that candidate lives in.

- City: The City that candidate lives in.

- Work Status: Candidate is working or not. Divide in 2 groups: True or False. 
- Employment Status: The information about the candidate's employment status in the course of application. Divide in 2 groups: True or False.

- Employment Preference: Candidate's employment preference. Divide in 5 groups: Full Time, Part Time, Volunteer, Intern and Project Based.

- Grades: Candidate's graduation degree.

- Foreign Languages: Candidate's knowledge of foreign language.

- Certificates: The information about the candidate's certificates.

- Experience: Total experience time of candidate.

- Business Area: The business fields of the candidate who preferred to work (IT, Reserach\&Development, financ, etc.).

- Sector: The sectors of the candidate who preferred to work (Informatics, health, media,etc.)

- Skills: Extra knowledge/skills possessed by the candidate.

\subsubsection{Candidate interaction features}

There are 9 more features in addition to those 19 features above in the data set used for the candidates. These features reflect the actions of the job announcing companies on the candidate's Curriculum Vitae (CV). These are as follows:

- CV Opened: CV is opened by the Employer or not.

- Drawer Thrown: CV is saved by the Employer to look again or not.

- Short Long List: CV url added to the list by the Employer or not.

- Printed CV: CV is printed by the Employer or not.

- CV Shared: CV is shared with others by the Employer or not.

- Job Offered: Candidate has been offered by employer or not.

- Interview: Candidate was invited to interview or not.

- CV Multiple Times Opened: CV is looked more than once or not.

\subsubsection{Job profile features.}

- Job Location: After getting the job, where the candidate will be working.

- Position Offered: Position offered for the job.

- Employer/Company: Company that is offering the job.

- Sector: major/discipline in which the job is offered.

- Experience: Required experience for the job.

- Education: Required education for the job.
- Military Situation: Required military situation for the job.

- Certificate: Required certificates for the job.

- Foreign Language: Required foreign language information for the job.

- Requirements: Required extra knowledge/skills for the job.

\subsection{Problem Definition}

The main problem in reciprocal job recommendation systems is to make a match that fulfils the mutual needs of both users (candidate and job). In these systems, the relations between the users must be modeled considering the preferences of both sides. In job recommendation systems, the job seekers look for positions that match their preferences and the employers search suitable candidates to fulfill the requirements of the job. A reciprocal job recommendation scenario consists of two separate set of users, C and J users to represent the set of candidates and jobs, depending on $\mathrm{c} \in \mathrm{C}$ and $j \in J$, the job list $R 1(c)$ recommended for $c$ candidate, the candidate list $\mathrm{R} 2(\mathrm{j})$ recommended for $\mathrm{j}$ job, to match the $(\mathrm{c}, \mathrm{j})$ couple reciprocally, $\mathrm{c}$ and $\mathrm{j}$ users need to be in each other's preference list [2]. In formula 1 JRR represents job reciprocal recommendation.

$$
R R:\{j: j \in R 1(c) \cap c \in R 2(j)\}
$$

Where, RR: Reciprocal Recommendation.

In this study, a method is proposed to recommend jobs in an order, which candidate may prefer and which the candidate may be in the jobs preference list, to a new candidate signed up to the system in job recommendation systems. At the same time, this method avoids candidates' cold-start problem and creates solutions for data sparsity problem caused by uncertainty of the answers to the applications in the dataset.

\section{Framework Overview}

Figure 1 illustrates the CCRS general working structure of the reciprocal job proposal system. The recommended system consists of 4 components that are associated with each other. There is information on the candidates in the dataset taken from [10] on which jobs they are applying, but there is no information on the positive/negative feedbacks to the candidates on the jobs. In order to address this deficiency in the dataset, two different methods of determining the return values of the applications specified in section 5 have been proposed. Two different studies have been carried out by applying the four basic steps of the proposed model to the label information obtained from the two methods due to the different label information obtained for these 
applications. Only the label determination parts of these two studies are different from each other, and the operations performed in 4 steps after this step are the same. Steps of the proposed methods;

\subsection{Creation of the Job List to be Proposed to The Candidate}

In order to make a recommendation to a candidate who is entering the system recently, the first $\mathrm{n}$ of the candidates similar to this candidate are to be found and a CandidateList is to be created. If the candidate is not a cold start, then this candidate himself/herself is added to the CandidateList as well. Afterwards, the previous jobs to which the candidates of the CandidateList applied are found and the JobList is created. Additionally, the first $\mathrm{m}$ of the jobs similar to the jobs in the JobList are added to the JobList. The list of the jobs which the target candidate may prefer to apply to is obtained in this way. In order to calculate the similarity between the users (candidate, job) with the users within their own set the cosine similarity formula is used. Cosine similarity between 2 vectors a \& b is calculated by the following formula:

$$
\text { Cosine similarity }(\mathrm{a}, \mathrm{b})=(\mathrm{a} \cdot \mathrm{b}) /(|\mathrm{a}||\mathrm{b}|)
$$

These $\mathrm{m}$ and $\mathrm{n}$ values have been obtained as a result of the trials conducted on a validation set consisting of applications. As a result of these experiments the $\mathrm{n}$ and $\mathrm{m}$ values have been found in the study with user behavior to be $\mathrm{n}: 10 \%, \mathrm{~m}: 1 \%$, and in the study with Trust Rank method to be n: $15 \%, \mathrm{~m}: 3 \%$.

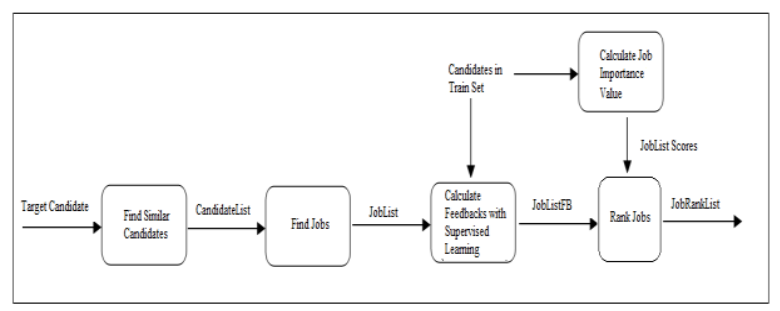

Figure 1. An overview of CCRS

\subsection{Obtaining The Return Values of The Jobs}

The label value indicating the return value of all jobs in the data set that replied to all candidates from whom they received an application is obtained, by using the interactions on the curriculum vitae of the candidates who previously applied to the jobs. These label values are trained for each job via methods of classification and the reply of the jobs in the JobList that may be sent to the target candidates is calculated in this way. The target candidate feedback value of the jobs has been designated as FB. In this way, the information whether they will be preferring the target candidate for each job in the JobList is obtained (JoblistFB).

\subsection{Determining the Importance Values of the Jobs}

A confidence value is calculated for each candidate by using the applications in the system that were previously carried out by the candidates. The confidence value is the value obtained from the similarity of the jobs to which a candidate has applied to. If the similarity among the jobs the candidate applied to is very high, the confidence value of the candidate is high, if it is low then the confidence value of the candidate is low. Each candidate provides his/her confidence value to the job they apply to. The importance value of the jobs is determined through the sum of the candidates who are applying to it and these importance values are accepted as the score value of the jobs and names as $\mathrm{SV}$. Thus, a score value is obtained for each job in the JobList (JobListScore).

\subsection{Creation of The Ranked Job Recommendation List}

The FB value and SV values of each job in the JobList are multiplied and the jobs are recommended to the target candidates in accordance with the obtained values, thus obtaining the JobRankList.

\section{Methods Used in The Proposed Model}

In this section the method used in the proposed model are described.

\subsection{Determination of the Return Values of the Jobs Related to The Applications}

There is information on the candidates in the dataset taken from [10] on which jobs they are applying, but there is no information on the positive/negative feedbacks to the candidates on the jobs. In this section, explanations of the two labeling methods proposed for eliminating this data sparsity are made. The label values obtained for these applications from these methods are used as the actual feedback values of the jobs, and are used in the evaluation section to calculate the successful of the proposed model as the actual label values.

5.1.1. Label identification method with user behavior. In the first proposed method, it is aimed to find candidates for which the companies would like to hear from the interactions information of the candidate in order to decide on the responses to the applications. If the job announcing company takes 
action for at least 4 on the $\mathrm{CV}$ of the candidate applying for the job, these candidates are assumed to be hired for the job and these applications are labeled with the "1" class whereas the opposite cases are labeled with "0". As a result of these operations label 1 is used for 748 of applications in total.

5.1.2. Label identification method with Trust Rank method. In the previous section, when label information and application information obtained using the candidates' interactions information were examined, it was seen that most of the applications were mislabeled. For this reason, Trust Rank[11] method, which is a semantic method and used for detecting unwanted users, has been utilized in the second method for identification of labels. Some definitions in the Trust Rank model have been changed so that the Trust Rank method can be applied on the dataset that is used. According to these changes, the applications made up the nodes on the graph, the cosine similarity between the applications was taken as the transition matrix, and the links were made among the people who applied to the same jobs by creating a separate graph within 10 jobs. Thus, 10 separate graphs and 10 separate seed sets have been created for 10 different jobs. For each graph, the seed set consists of the label values of the applications. -1 value represents application rejected, +1 value represents application accepted, and 0 value represents application result unknown.

With these label values, random applications from both classes are selected and checked whether they have the correct labels and then seed sets are obtained with the applications which have the right labels. Applying the Trust Rank method to the dataset resulted in a total of $1967+1$ labeled applications.

\subsection{Obtainment of The Confidence Values of The Candidates and The Importance Values of The Jobs}

The confidence values of the candidates and the importance values of the jobs are being used for the determination of the place of the recommended jobs that have been recommended to the candidates. The confidence value of the candidates determines the similarity among the applied jobs. If the candidates apply to dissimilar jobs then the confidence value is high. The confidence value of the candidates is determined by the sum of the similarity values among the jobs that have been applied to by the candidates as in formula 3 .

$$
\operatorname{AGD}(\mathrm{u})=\frac{1}{\sum_{\mathrm{f}=1}^{\mathrm{m}-1}(\mathrm{~m})} \sum_{\mathrm{i}=1}^{\mathrm{m}-1} \sum_{\mathrm{j}=\mathrm{l}+1}^{\mathrm{m}}\left(\operatorname{sim}\left(\mathrm{j}_{\mathrm{j}}, \mathrm{j}_{\mathrm{i}}\right)\right)
$$

Where, $\operatorname{AGD}(\mathrm{C})$ : Confidence value of candidate $\mathrm{C}$,
$\operatorname{Sim}(\mathrm{Ji}, \mathrm{Jj}):$ Similarity value between the $\mathrm{i}$ and j jobs

m: Number of jobs candidate $\mathrm{C}$ applied to,

The importance value of the jobs is obtained via the sum of the confidence values of the candidates that applied for the job as in formula 4.

$$
\operatorname{IOD}(\mathrm{J})=\sum_{c=i}^{n} \operatorname{AGD}(\mathrm{c})
$$

Where, $\operatorname{IOD}(\mathrm{J})$ : Importance value of the $\mathrm{J}$ job, AGD: Confidence value of candidate $C$, $\mathrm{n}$ : Number of candidates applying for the $\mathrm{J}$ job.

\section{Experimental Evaluation}

\subsection{Data Acquisition}

In order to measure the performance of the proposed method, data set obtained from Kariyer.Net[10] website has been used. In this data set, candidates and job postings consist of qualities specified in Section 3. Data set consists of 7455 applications in total to 10 job postings from 5600 candidates, and includes only job postings issued for software engineering positions. Label information obtained in Section 5 have been used to measure the success of methods. For assignment of the parameters of the classification methods used in estimating the feedback values of jobs, finding similarities of candidates and jobs and the feature selection of candidates a validation set consisting of 1000 applications was used. Accuracy of the method was measured for both cold start and non cold start candidates by creating two evaluation sets.

Excluding the 1000 applications used in determining parameters, both evaluation sets were divided into two sets as train and test. Accordingly, train set for cold start candidates consisted of 3216 applications from 1336 candidates who made at least 2 applications, while test set consisted of applications made by 3239 candidates with just 1 application. Test set was divided into three equal pieces, and the average of success values in these pieces was calculated. For non cold start candidates, train and test sets were created by dividing 3216 applications, made by candidates with at least 2 applications, into two. Accordingly, education set consists of 2145 applications, and test set consists of 1071 applications.

\subsection{Evaluation Metrics}

Proposed method was programmed with java, using weka library [12] for classification methods. Two measurements are used for evaluating the success of the proposed method. 


\subsubsection{Ranking evaluation}

Mean averaged precision (map) measurement is used to measure the accuracy of recommendation ranks of applications made by the candidates, and the candidates were accepted to. Methods used for this are precision@k and recall@k. These methods generate a success value according to the rank of actually recommended applications on the top-k recommendation list [13].

$$
\text { ap@ } \mathrm{q}=\sum_{\mathrm{i}=1}^{\mathrm{Q}} \mathrm{P}(\mathrm{k}) / \min (\mathrm{r}, \mathrm{q})
$$

Where, $\mathrm{P}(\mathrm{k})$ : Precision value for first $\mathrm{k}$ number of applications proposed,

r: Number of applications that should be actually proposed,

q: Number of actually proposed applications on the recommendation list.

After average precision values for each candidate on the recommendation list are obtained, mean average precision is used to calculate precision value of the recommendation queues for each candidate. This method takes the average of average precision values obtained for each candidate.

$$
\text { MAP@q }=\sum_{\mathrm{i}=1}^{\mathrm{Q}} \mathrm{ap} @ \mathrm{qi} / \mathrm{Q}
$$

Where, Q: Candidate number

i: Candidate's precision value

\subsubsection{Evaluation of accuracies}

True negative rate (TNR) measurement is used to calculate the accuracy of applications that are actually made by the candidate and the candidate was rejected are not on the recommendation list[13]. For candidate $\mathrm{C}$, actual rejected applications $\mathrm{M}$, and applications not recommended to the candidate $\mathrm{N}$, TNR is calculated as following;

$$
\mathrm{TNR}=\frac{\mathrm{M} \cap \mathrm{N}}{\mathrm{M}}
$$

\subsection{Experimental Results}

In this section, the effects of the classification methods on the accuracy of the proposed method has been evaluated and the proposed method has been compared to previously proposed methods. Classification methods used for the proposed method are Naive Bayes (NB), Support Vector Machine (SVM) with rbf kernel classifier, Multilayer Perceptron (MLP) and C4.5. Detailed descriptions of these classification methods can be found in [14].

The methods used to compare the proposed method are content-collaborative reciprocal recommender (CCR) [6] and a mobile reciprocal job recommender system (iHR+) [8]. CCR is a reciprocal content-collaborative recommendation system proposed for online dating systems. The content-based part allows similar users to be found by user profiles, and the collaborative filtering part produces reciprocal recommendation using interactions of similar users. Using similar users to provide recommendations, and producing recommendations among cold start users, CCR shows similarities with the proposed CCRS system.

Therefore, CCR can be defined as a special condition of the CCRS system. iHR+ is a mobile application system that provides reciprocal job recommendations. In this method, attributes of job seekers and recruiters are separated into two categories: self-description and preferences. The similarity between these attributes is calculated by cross-similarity and reciprocal recommendations are generated. Since the iHR+ method uses profile information of users to make recommendations and also considers data sparsity, it is a method that can be applied to the CCRS system. Therefore it can be compared to the proposed CCRS method.

6.3.1. Evaluation results of the work created by user behavior. In this section, there are the results obtained for the generated model with the label values obtained using the interaction features of the candidates. The resulting parameter values for the classification methods used are: for SVM complexity $\operatorname{parameter}(\mathrm{c})=10$, kernel parameter $(\gamma)=0.07$; for MLP $\operatorname{seed}(s)=3$, hiddenLayers $(h)=10$; for $\mathrm{C} 4.5$ confidenceFactor $=0.01$.

In Table 1, different classification methods for the proposed method on the evaluation set generated for cold start candidates, and MAP and TNR results obtained for the compared methods are shown. According to this, the best TNR value for the cold start data set is obtained by MLP, while the best MAP value is obtained by $\mathrm{C} 4.5$. The proposed method has achieved higher success for MAP and TNR from CCR and iHR+ methods, for all classification methods tried.

In Table 2, the results obtained from non cold start candidates are shown. According to this, the best TNR value is obtained by MLP, while the best MAP value is obtained by SVM. For both data sets, the TNR value for the iHR+ method is zero. This is because all applications are proposed to the candidates since the similarity between candidates and 10 applications are all higher than zero.

Table 1. MAP and TNR values of compared methods performance for cold start data set using with interaction features

\begin{tabular}{|l|l|l|l|l|l|l|}
\hline & NB & C4.5 & MLP & $\begin{array}{l}\text { RBF } \\
\text { SVM }\end{array}$ & CCR & iHR+ \\
\hline MAP & 0.42 & $\mathbf{0 . 4 9}$ & 0.43 & 0.48 & 0.43 & 0.37 \\
\hline TNR & 0.56 & 0.55 & $\mathbf{0 . 5 7}$ & 0.56 & 0.48 & 0.04 \\
\hline
\end{tabular}


Table 2. MAP and TNR values of compared methods performance for non cold start data set using with interaction features

\begin{tabular}{|l|l|l|l|l|l|l|}
\hline & NB & C4.5 & MLP & $\begin{array}{l}\text { RBF } \\
\text { SVM }\end{array}$ & CCR & iHR+ \\
\hline MAP & 0.52 & 0.51 & 0.48 & $\mathbf{0 . 5 6}$ & 0.48 & 0.47 \\
\hline TNR & 0.58 & 0.57 & $\mathbf{0 . 6 1}$ & 0.53 & 0.45 & 0 \\
\hline
\end{tabular}

In Figure 2 and Figure 3, precision values of the methods for cold start and non cold start data sets are shown. For every $\mathrm{k}$ value in the cold start data set, similar precision values were obtained for both $\mathrm{C} 4.5$ and SVM classification methods. For every k value in the non cold start data set, the best result was obtained for the SVM method.

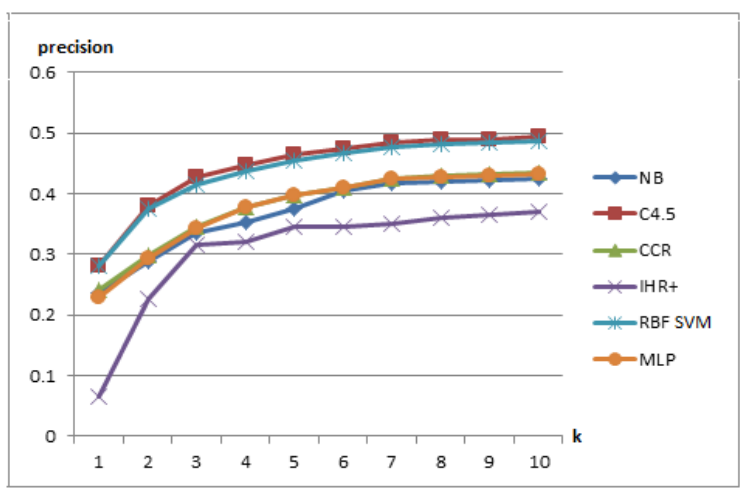

Figure 2. Precision@k for cold start data set using with interaction features

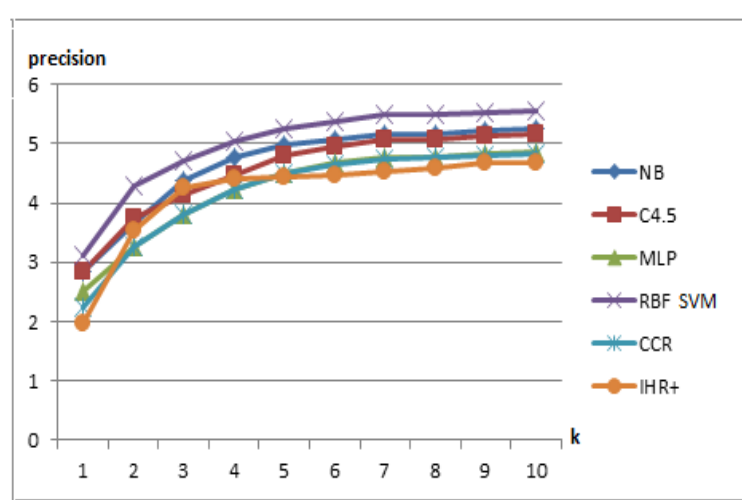

Figure 3. Precision@k for non cold start data set using with interaction features

According to these results, SVM method can be used in this data set for MAP evaluation, since C4.5 and SVM values are very similar for the MAP values obtained from the cold start data set, and SVM method is significantly better than the remaining methods in the non cold start data set. For TNR, however, MLP obtained the highest values for both data sets.

6.3.2. Evaluation results of the work created by Trust Rank method. In this section, the results obtained for the model using the labels obtained by the Trust Rank method are available. Unlike the previous work, in obtaining the return values of the jobs section, the features obtained by using information gain feature selection method[15] were used. The parameter values of the classification methods used for this model are: for SVM complexity parameter $(\mathrm{c})=3$, kernel $\operatorname{parameter}(\gamma)=$ 0.01; for MLP seed(s)=3, hiddenLayers $(h)=10$; for C4.5 confidenceFactor $=0.1$.

Table 3 shows the MAP and TNR results for the data set consisting of cold-start candidates. According to these results, the best TNR and MAP values for this data set is obtained by RBF SVM, and higher success rates for MAP and TNR metrics were obtained from CCR and iHR+ methods.

Table 4 shows the MAP and TNR results obtained with the evaluation set formed with noncold-start candidates. Accordingly, the best MAP and TNR values for the data set consisting of noncold-start candidates were obtained with RBF SVM. RBF SVM method provides higher success for MAP and TNR than CCR and iHR+ methods in this data set, as it is in the data set consisting of cold-start candidates.

Table 3. MAP and TNR values of compared methods performance for cold start data set using with Trust Rank method

\begin{tabular}{|l|l|l|l|l|l|l|}
\hline & NB & C4.5 & MLP & $\begin{array}{l}\text { RBF } \\
\text { SVM }\end{array}$ & CCR & iHR + \\
\hline MAP & 0.69 & 0.72 & 0.73 & $\mathbf{0 . 7 4}$ & 0.70 & 0.65 \\
\hline TNR & 0.82 & 0.83 & 0.83 & $\mathbf{0 . 8 5}$ & 0.83 & 0.07 \\
\hline
\end{tabular}

Table 4. MAP and TNR values of compared methods performance for non cold start data set using with Trust Rank method

\begin{tabular}{|l|l|l|l|l|l|l|}
\hline & NB & C4.5 & MLP & $\begin{array}{l}\text { RBF } \\
\text { SVM }\end{array}$ & CCR & iHR+ \\
\hline MAP & 0.60 & 0.73 & 0.74 & $\mathbf{0 . 7 6}$ & 0.67 & 0.60 \\
\hline TNR & 0.90 & 0.91 & 0.85 & $\mathbf{0 . 9 3}$ & 0.87 & 0.07 \\
\hline
\end{tabular}

Figures 4 and 5 show the precision values of the methods in the ' $k$ 'th step for the two data sets. The highest result for each $\mathrm{k}$ values in the data set consisting of cold start problem candidates was found by the RBF SVM method. For candidates with non cold start problem, close values were obtained both C4.5 and RBF SVM classification methods with $\mathrm{k}=1$, and the highest result for the other $\mathrm{k}$ values was found by the RBF SVM method.

According to these results, RBF SVM can be used for the MAP and TNR evaluation in this data set, because the RBF SVM method is significantly higher than the other methods in the two data sets for the MAP and TNR values obtained by using the Trust Rank. 


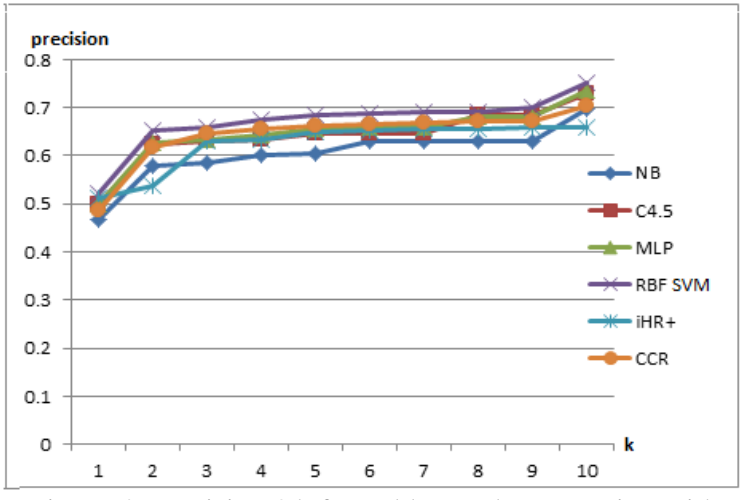

Figure 4. Precision@k for cold start data set using with Trust Rank method

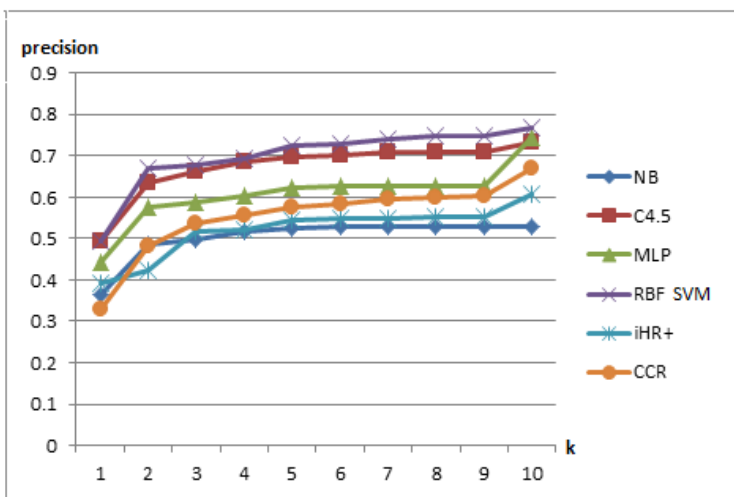

Figure 5. Precision@k for non cold start data set using with Trust Rank method

\section{Conclusion}

In this study, a reciprocal business recommendation system (CCRS) is proposed. In order to recommend jobs to the candidates, the proposed system takes advantage of applications of similar candidates, and preference information on jobs. Created system has been tested using different classification methods. Two labeling methods have been proposed to eliminate the problem of sparse data that is originating from the lack of answer information in the data set used, and two different studies have been conducted to compare the effects of both labeling methods on the dataset. When the results obtained are examined, it is observed that the results of the study using Trust Rank in general are higher than those of both the results of the study using user behavior and the other two methods that were compared (iHR + and CCR) for MAP and TNR metrics.

The reason for the better results of the study created by using the Trust Rank is that the method of labeling with Trust Rank is a method which is formed by the purpose of eliminating the deficiencies created by using the data generated via labelling by user behavior, and that the label values obtained from the methods are different and due to the fact that the parameter values obtained for the proposed model are different for both studies, it is considered that the accuracy obtained by the Trust Rank generated study is better. According to the results of this evaluation, it is accepted that the best achievement for MAP and TNR measurement using the Trust Rank method for labeling in the proposed model is obtained by the RBF SVM classification method. As part of our future studies, plans are being made on assessment of the proposed method on bigger data sets.

\section{References}

[1] Zhao, K., Wang, X., Yu, M., Gao, B. (2014). User Recommendations in Reciprocal and Bipartite Social Networks--An Online Dating Case Study. IEEE Intelligent Systems, 29 (2), $27-35$.

[2] Pizzato L., Rej T., Chung T., Koprinska I., Yacef K. \& Kay J. (2010). RECON: a reciprocal recommender for online dating. Proceedings of the fourth ACM conference on Recommender systems, (pp.207-214). ACM, New York, NY, USA, September $26-30$.

[3] Pazzani, M.J. \& Billsus, D. (2007). Content-based recommendation systems. The Adaptive Web, 4321, 325 341.

[4] Konstan, J. A., Miller, B. N., Maltz, D., Herlocker, J. L., Gordon, L. R., \& Riedl, J. (1997). Applying Collaborative Filtering to Usenet News. Communications of the ACM, 40 (3), 77-87.

[5] Burke, R. (2002). Hybrid recommender systems: Survey and experiments. User Modeling and User-Adapted Interaction, 12 (4), 331-370.

[6] Akehurst, J. , Koprinska, I., Yacef, K. , Pizzato, L., Kay, J. \& Rej, T. (2011). Ccr-a content-collaborative reciprocal recommender for online dating. Proceedings of the Twenty-Second international joint conference on Artificial Intelligence, (pp.2199-2204). AAAI Press, July $16-22$.

[7] Adomavicius, G., Tuzhilin A. (2005). Toward the next generation of recommender systems: a survey of the stateof-the-art and possible extensions. IEEE Transactions on Knowledge and Data Engineering, 17(6), 734-749.

[8] H. Wenxing, C. Yiwei, Q. Jianweiand H. Yin (2015). iHR+: A Mobile Reciprocal Job Recommender System. 10th International Conference on Computer Science \& Education (ICCSE), $492-495$.

[9] N. D. Almalis, G. A. Tsihrintzis, N. Karagiannis (2014). A content based approach for recommending personnel for job positions. 5th International Conference on Information, Intelligence, Systems and Applications (IISA).

[10] Kariyer.net Available: www.kariyer.net

[11] Krestel, R. and Chen, L. (2008). Using co-occurence of tags and resources to identify spammers, ECML PKDD Discovery Challenge. 
[12] (2008). Weka Machine Learning Project, University of Waikato, http://www.cs.waikato.ac.nz/ml/weka/.

[12] Schein, Andrew I., Popescul, Alexandrin, Ungar, Lyle H., \& Pennock, David M. (2002). Methods and Metrics for Cold-Start Recommendations. Proceedings of the 25th Annual International ACM SIGIR Conference on Research and Development in Information Retrieval, (pp.253-260). ACM, New York, NY, USA, August $11-15$.

[14] Alpaydin, E., (2010). Introduction to Machine Learning, The MIT Press, Cambridge, Massachusetts London, England, 2. edition.

[15] Tang, Jiliang and Alelyani, Salem and Liu, Huan, (2014). Feature selection for classification: A review. In: Data Classification: Algorithms and Applications. CRC Press. 